\title{
MÉTODO ALTERNATIVO DE AVALIAÇÃO DA PRESSÃO DE PRECONSOLIDAÇÃO POR MEIO DE UM PENETRÔMETRO'(1)
}

\author{
M. S. DIAS J UNIOR ${ }^{(2)}$, A. R. SILVA(3), S. FONSE CA ${ }^{(4)} \&$ F. P. LEITE ${ }^{(5)}$
}

\begin{abstract}
RESUMO
A curva de compressão do solo tem si do freqüentemente utilizada em estudos de compactação do solo. Essa curva representa graficamente a relação entre o logaritmo da pressão aplicada e a densidade do solo ou o índice de vazios. A pressão de preconsolidação divide essa curva em uma região de deformações recuperáveis e em uma de deformações não-recuperáveis. Por esse motivo, a pressão de preconsolidação tem sido usada como uma estimativa da capacidade de suporte de carga dos solos parcialmente saturados. A pressão de preconsolidação é obtida em laboratório por meio do ensaio de compressão uniaxial, o qual requer aparelhos sofisticados para a sua realização. Além do tempo gasto na realização do ensaio, há necessidade de usar um método gráfico ou de planilhas para a sua determinação. Uma maneira alternativa que visa minimizar este problema seria a cali bração da pressão de preconsolidação com outras medidas de fácil e rápida obtenção. 0 objetivo deste trabalho foi propor um método alternativo de avaliação da pressão de preconsolidação por meio de um penetrômetro. Para isso, foram determinadas as pressões de preconsolidação e as resistências à penetração, no laboratório, na camada superficial de um Argissolo Amarelo (PA) e de um Plintossolo (FX) localizados na regia ão de Aracruz, ES. Testes de campo foram efetuados em um Latossolo Vermelho-Amarelo (LV) localizado em Belo Oriente, MG. Os valores dos coeficientes de determi nação foram significativos e variaram de 0,88 a 0,99. As equações que relacionam a pressão de preconsolidação $\left(\sigma_{p}\right)$ com a resistência à penetração $(R P)$ foram da forma: $\sigma_{p}=a+b(R P)$. Pela equação, as pressões de preconsolidação serão estimadas em função da resistência à penetração e poderão ser utilizadas na identificação da compactação do solo e como uma ferramenta auxiliar de deci são sobre a realização ou não de determi nada operação mecanizada.
\end{abstract}

Termos de indexação: compactação do solo, resistência à penetração, pressão de preconsolidação, modelagem.

(1) Recebido para publicação em outubro de 2000 e aprovado em agosto de 2004.

(2) Professor Adjunto do Departamento de Ciência do Solo, Universidade Federal de Lavras. Caixa Postal 37, CEP 372000-000 Lavras (MG). Bolsista do CNPq. E-mail: msouzadj@ufla.br

(3) Aluno de Iniciação Científica. Bolsista do CNPq.

(4) Engenheiro Florestal, Aracruz Celulose S.A. Caixa Postal 331011, CEP 29197-000 Aracruz (ES). E-mail: sf@aracruz.com.br

(5) Engenheiro-Agrônomo, Celulose Nipo-Brasileira S.A. Caixa Postal 100, CEP 35160-970 Ipatinga (MG). E-mail: fernandoleite@cenibra.com.br 


\title{
SUMMARY: ALTERNATIVE METHOD FOR THE EVALUATION OF THE PRECONSOLIDATION PRESSURE THROUGH A PENETROMETER
}

\begin{abstract}
Soil compression curves arefrequently used in compaction studies. Thesecurves describe therelati onship between thelogarithm of applied pressureand thesoil bulk density or void ratio. The preconsolidation pressure divides the soil compression curves into a region of recoverableand a region of unrecoverabledeformations. Consequently, thepreconsol idation pressure has been used as an estimate of soil load support capacity of partially saturated soils. Thepreconsol idation pressureis laboratory-obtained through theuniaxial compression test which requires sophi sti cated equi pment. Besides the timespent on the test realization, it is necessary to use a graphic method or spreadsheet procedure for its determination. An alternative way to mi nimize this problem would be the calibration of the preconsolidation pressure by other ways of easy and fast achievement. Thus, the objective of this study was to proposean alternativeevaluation method of the preconsol idation pressureby a penetrometer. F or this purpose, thepreconsolidation pressures and penetration resistancein thesuperficial layer of a Yellow Argisol (PA) and Plinthosol (FX) from Aracruz (ES), B razil, weredetermined in thelaboratory. Field tests were carried out with an Oxisol (LV) located in Belo Oriente (MG). The coefficients of determination weresignificant and varied from 0.88 to 0.99 . The equations that relatethe preconsol idation pressure $\left(\sigma_{\mathrm{p}}\right)$ with the penetrati on resistance (RP) were of theform: $\sigma_{\mathrm{p}}=\mathrm{a}+\mathrm{b}$ (RP). This equation permits theestimation of thepreconsolidation pressureas a function of thepenetrometer resistance, which may beused in theidentification of the soil compaction and as a tool to come to a decision about realizing a particular mechanized operation or not.
\end{abstract}

Index terms: Soil compaction, penetration resistance, preconsolidation pressure, modeling.

\section{NTRODUÇÃO}

Recentes pesquisas na área de compactação do sol o têm dado ênfase ao desenvol vimento de model os matemáticos para a previsão da compactação dos sol os (DiasJ unior, 1994; Dias J unior \& Pierce, 1996; Kondo \& Dias J unior, 1999, Gysi, 2001; Canillas \& Salokhe, 2002; Defossez \& Richard, 2002). Um dos enfoques dessa modelagem tem considerado a pressão de preconsolidação do solo como sendo a propriedade que expressa a capacidade de suporte de carga dos solos parcialmente saturados (Kondo \& Dias J unior, 1999; Dias J unior et al., 1999).

A pressão de preconsolidação é obtida em laboratório por meio das curvas de compressão do solo (Vargas, 1977; Holtz \& Kovacs, 1981, Kondo \& Dias J unior, 1999), as quais são obtidas pelo ensaio de compressão uniaxial, o qual requer equipamentos sofisticados para a sua realização. Além do tempo gasto nesses ensaios, há necessidade de adotar métodos, tais como os propostos por Casagrande (1936), Burmister (1951), Schmertmann (1955), Sällfors (1975), Anderson \& Lukas (1981), Culley \& Larson (1987), J osé et al. (1989), Lebert \& Horn (1991), Dias J unior \& Pierce (1995), para a sua determinação.

Apesar de estar o método para a determinação da pressão de preconsolidação difundido (Vargas, 1977; Holtz \& Kovacs, 1981; Bowles, 1986), são necessári os outros métodos al ternativos que possam ser utilizados no campo. U ma alternativa para a sua determi nação seria a cal i bração de seus val ores com outras medidas de fácil erápida obtenção no campo.

O objetivo deste trabal ho foi propor um método alternativo deavaliação da pressão de preconsolidação por meio de um penetrômetro.

\section{MATERIAL E MÉTODOS}

Neste estudo, foram utilizados um Argissolo Amarelo (PA) e um Plintossolo (FX), localizados em áreas experimentais da região de Aracruz, ES. Para realizar os ensaios de compressão uniaxial, foram col etadas aleatoriamente na área sob eucalipto 27 amostras indeformadas na profundidade de 0 0,03 m (superfície do horizonte A) e de 0,35-0,38 m (topo do horizonte B), totalizando 54 amostras para cada classe de solo. Estas amostras foram usadas no ensaio de compressão uniaxial (Bowles, 1986; Dias J unior, 1994, Kondo \& Dias J unior, 1999). No restante destas amostras, determinaram-se a textura (Day, 1986) e matéria orgânica (Raij \& Quaggio, 1983) (Quadro 1). A área em estudo já havia sido plantada por dois ciclos, eas amostragens aconteceram ao final do terceiro ciclo, sendo a idade do povoamento de nove anos. 
Para obter os modelos $\sigma_{p}=\mathrm{f}(\mathrm{U})$, os ensaios de compressão uniaxial foram realizados em amostras com diferentes umidades, as quais foram, inicialmente, saturadas e, a seguir, secas ao ar no laboratório até se obter a umi dade variando de 0,05 a $0,35 \mathrm{~kg} \mathrm{~kg}^{-1}$. Obtidas essas umi dades, as amostras foram submetidas ao ensaio de compressão uniaxial, usando-se um consolidômetro da marca Boart Longyear. As pressões aplicadas a cada amostra obedeceram à seguinte ordem: $25,50,100,200,400$, 800 e $1.600 \mathrm{kPa}$. Cada pressão foi aplicada até que 90 \% da deformação máxima fosseal cançada (Taylor, 1948), quando, então, foi aplicada uma nova pressão. Após o ensaio, as $\sigma_{\mathrm{p}}$ foram obtidas de acordo com Dias J unior \& Pierce (1995), usando as curvas de compressão do solo. A seguir, as $\sigma_{p}$ foram plotadas em função das umidades.

Próximo ao local de coleta das primeiras amostras, em ambos os solos e profundidades, foram col etadas mais 12 amostras indeformadas, nas quais se procedeu, em laboratório, à determinação da resistência à penetração (RP) em função da umi dade. Nestas determinações, utilizou-se um penetrômetro de bolso da marca SOI LTEST I nc., model o CL-700, com capacidade de leitura até $500 \mathrm{kPa}$.

As curvas de regressão entre $\sigma_{p}$ e $U$ e entre RP e $\mathrm{U}$ foram ajustadas, utilizando-se o software Sigma Plot 4.0 (J andel Scientific), enquanto a comparação das regressões foi feita segundo procedimento descrito por Snedecor \& Cocharan (1989).

Para avaliar a aplicação da equação $\sigma_{p}=f(R P)$ na identificação da compactação do solo, as pressões de preconsolidação de um Latossolo VermelhoAmarelo, na profundidade de 0,10-0,125 m, foram obtidas pela equação $\sigma_{p}=10^{(2,71-1,26 U)}, R^{2}=0,92 * *$, e as resistências à penetração pela equação

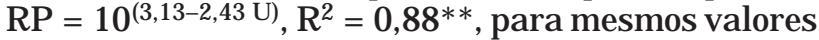
de umidade, o que permitiu a obtenção da equação $\sigma_{\mathrm{p}}=99,39+0,39 \mathrm{RP}, \mathrm{R}^{2}=0,99 * *$, conforme Dias J unior (2002). No campo, foram tomadas aleatoriamente seis determinações da resistência à penetração, em seis locais onde não houve tráfego de máquinas e em seis linhas de tráfego. Com a média das seis repetições, estimou-se a pressão de preconsolidação por meio da equação $\sigma_{p}=99,39+$ 0,39 RP a qual foi utilizada para identificar a compactação do solo.

\section{RESULTADOS E DISCUSSÃO}

As figuras 1 e 2 apresentam os modelos de sustentabilidade da estrutura para o PA e para o $\mathrm{FX}$, respectivamente, para as profundidades de 0 0,03 e de 0,35-0,38 m. Estes model os foram usados para obter as pressões de preconsolidação que foram plotadas em função da resistência à penetração obtida com o penetrômetro (Figura 5).

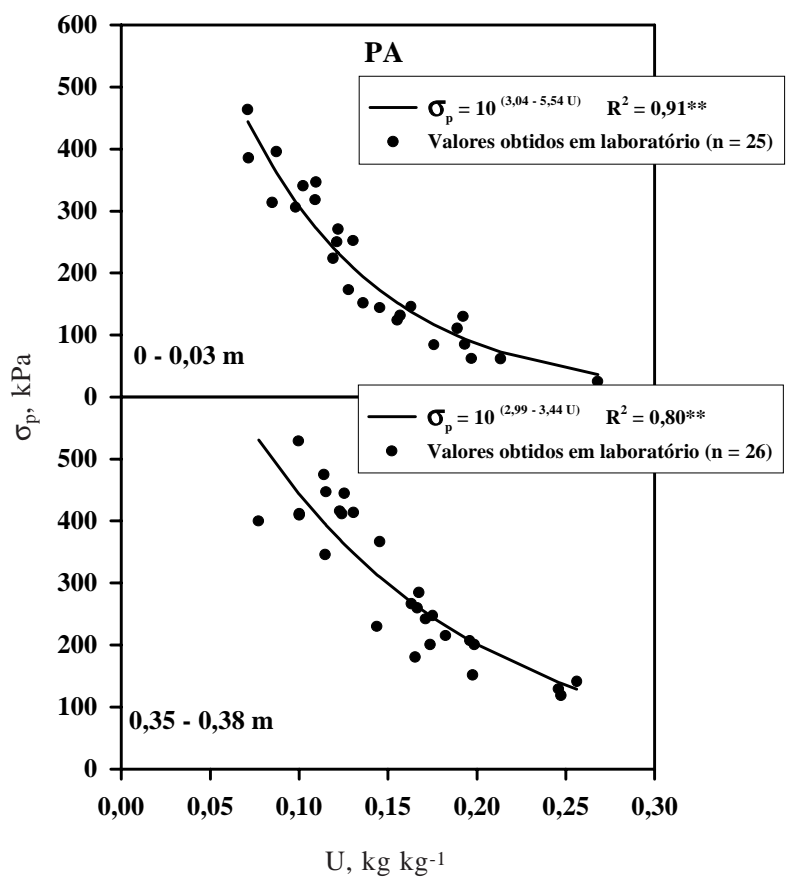

Figura 1. Modelos de sustentabilidade da estrutura para o PA, nas profundidades de 0-0,03 e de 0,35-0,38 m.

Quadro 1. Análises de caracterização física e de matéria orgânica (MO) de um Argissolo Amarelo (PA) e de um Plintossolo (FX), localizados em áreas experimentais da região de Aracruz, ES

\begin{tabular}{|c|c|c|c|c|c|}
\hline Classe de solo & Profundidade & Argila & Silte & Areia & MO \\
\hline & $\mathrm{m}$ & \multicolumn{4}{|c|}{$\mathrm{g} \mathrm{kg}^{-1}$} \\
\hline $\mathrm{PA}^{(1)}$ & $0-0,03$ & $190(3)$ & 87 & 723 & 14,0 \\
\hline PA & $0,35-0,38$ & 240 & 111 & 649 & 5,5 \\
\hline $\mathrm{FX}$ & $0-0,03$ & 130 & 75 & 795 & 31,2 \\
\hline $\mathrm{FX}$ & $0,35-0,38$ & 200 & 99 & 701 & 9,8 \\
\hline
\end{tabular}

(1) PA = Argissolo Amarelo, FX = Plintossolo. ${ }^{(3)}$ Média de três repetições. 


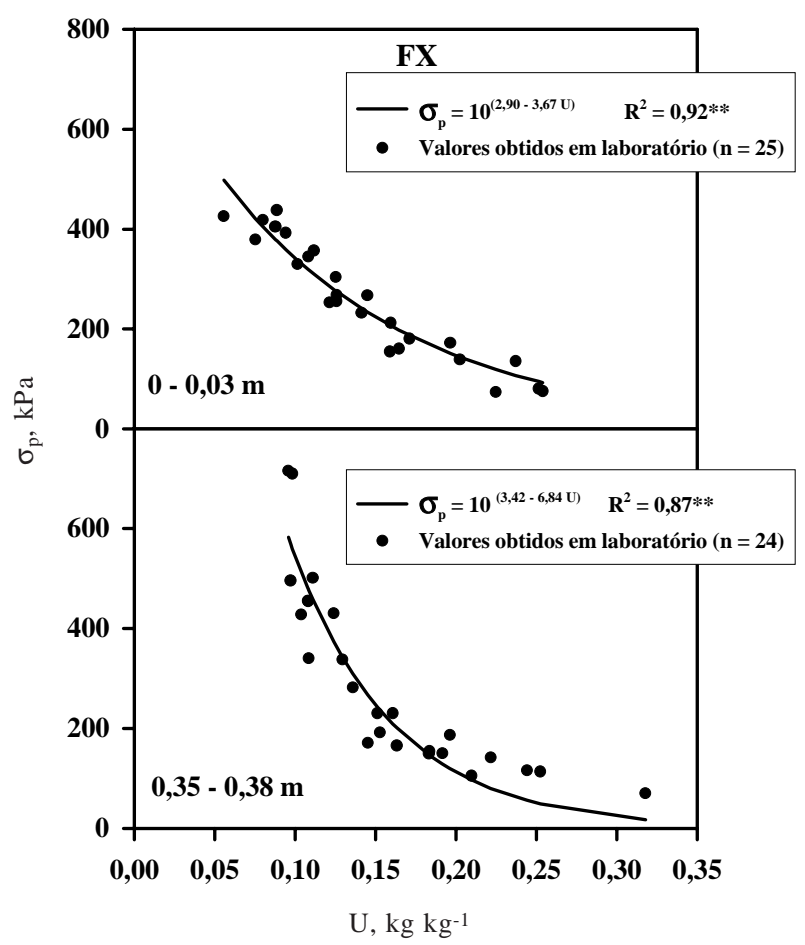

Figura 2. Model os de sustentabilidade da estrutura para o FX, nas profundidades de 0-0,03 e de $0,35-0,38 \mathrm{~m}$.

Os coeficientes de determinação das equações foram todos significativos e variaram de 0,80 a 0,87 (Figura 3). As equações para as profundidades de 0-0,03 e 0,35-0,38 m, em ambos os solos, foram estatisticamente diferentes, quando comparadas entre si, utilizando-se o procedimento descrito em Snedecor \& Cochran (1989). O mesmo ocorreu quando se compararam as equações para a profundidade de 0,35- 0,38 $\mathrm{m}$ para as duas classes de solo. Entretanto, as equações para a profundidade de $0-0,03 \mathrm{~m}$ do PA e do FX não foram estatisticamente diferentes, o que resultou na obtenção de uma única regressão para esta condição (Figura 4). Estas equações foram usadas para a obtenção da resistência à penetração que foram plotadas versus a pressão de preconsolidação (Figura 5).

A figura 5 apresenta as pressões de preconsolidação obtidas das equações apresentadas nas figuras 1 e 2 plotadas em função da resistência à penetração obtida das equações apresentadas na figura 4.

As equações que relacionam a pressão de preconsolidação $\left(\sigma_{p}\right)$ com a resistência à penetração (RP) foram da forma: $\sigma_{p}=a+b$ (RP) (Figura 5), concordando com os resultados obtidos por Dias J unior (1994). Estas equações foram todas estatisticamente diferentes pelo procedimento descrito em Snedecor \& Cochran (1989). Portanto, o PA e o FX nas profundidades estudadas apresentaram equações distintas para estimar a pressão de preconsol idação em função da resi stência à penetração.

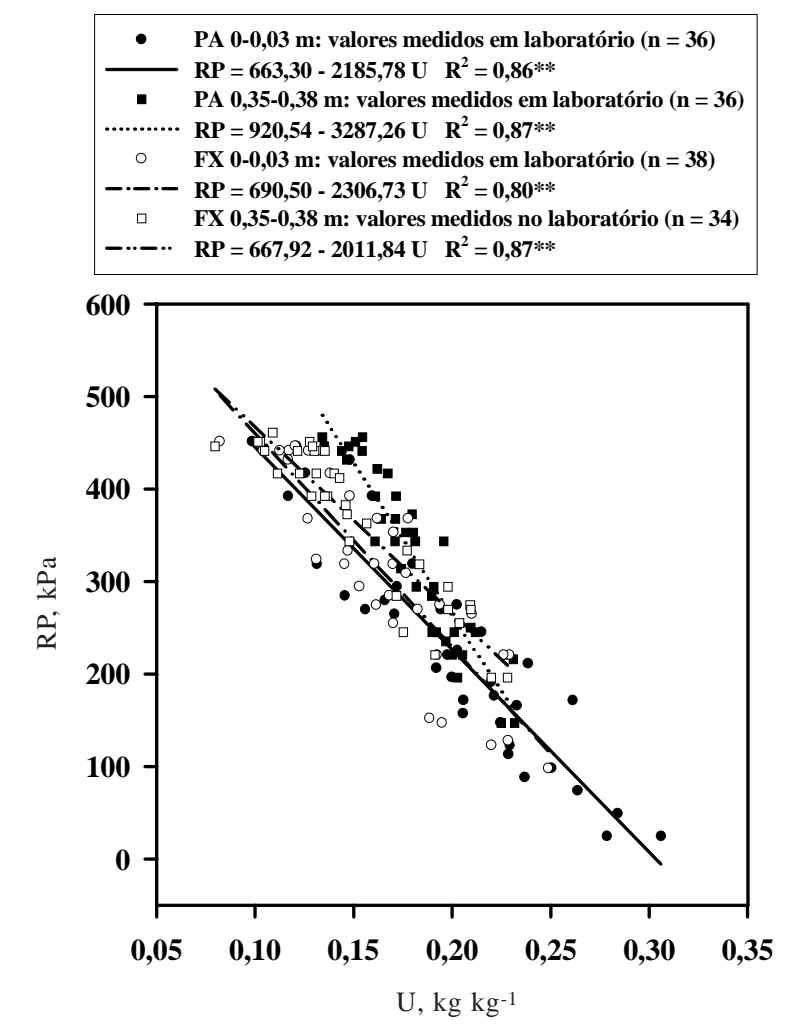

Figura 3. Resistência à penetração obtida com o penetrômetro no laboratório em função da umidade para o PA e para o FX, nas profundidades de 0-0,03 e de 0,35-0,38 $\mathrm{m}$.

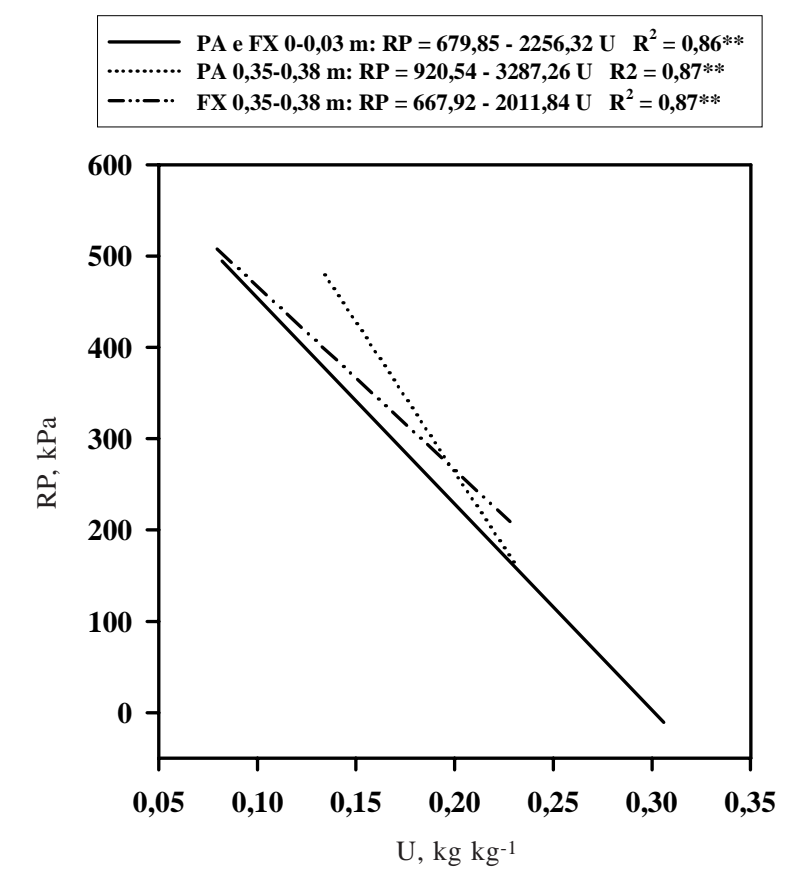

Figura 4. Resistência à penetração obtida com o penetrômetro no laboratório em função da umidade para o PA e para o FX, nas profundidades de 0-0,03 e de 0,35-0,38 m. 


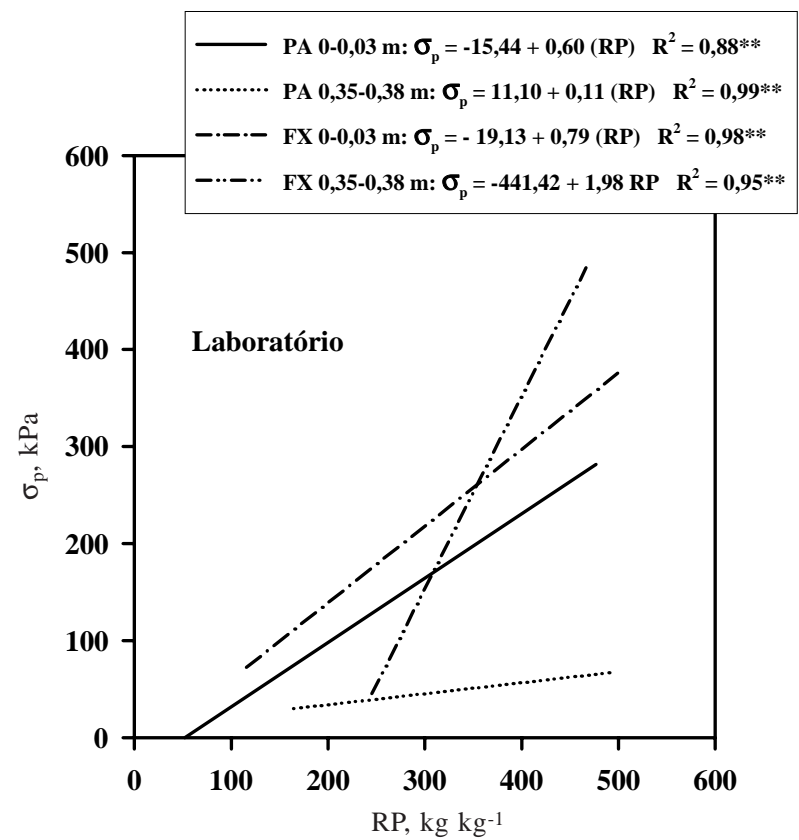

Figura 5. Pressão de preconsolidação em função da resistência à penetração para o PA e para o $F X$, nas profundidades de 0-0,03 e de 0,35$0,38 \mathrm{~m}$.

Por ser a resistência à penetração uma medida de fácil determinação no campo, o uso destas equações representará não só uma simplificação do método, mas também uma economia de tempo na determinação da pressão de preconsolidação e, portanto, da capacidade de suporte de carga do sol o no campo, a qual poderá ser usada como um critério auxiliar de decisão sobre o uso ou não das operações mecanizadas.

Para avaliar a aplicação da equação $\sigma_{p}=f(R P)$ na identificação da compactação do solo, as pressões de preconsolidação de um Latossolo VermelhoAmarelo, na profundidade de 0,10-0,125 m foram estimadas por meio da equação $\sigma_{p}=99,39+0,39$ $R P, R^{2}=0,99 * *$, conforme Dias J unior (2002). Considerando que, na agricultura, a aplicação de pressões maiores do que a maior pressão suportada pelo solo deve ser evitada, para que não ocorra a compactação adicional do solo (Gupta et al., 1989; Lebert \& Horn, 1991; I mhoff et al., 2001), e por constituir a pressão de preconsolidação um indicativo da máxima pressão aplicada ao solo no passado (Holtz \& Kovacs, 1981; Dias J unior, 1994), a figura 6 foi dividida em três regiões com vistas em identificar a compactação do solo por meio da pressão de preconsolidação estimada, conforme descrito anteriormente. Nesta figura, três regiões foram consideradas: (a) uma região onde as pressões de preconsolidação são maiores do que as pressões de preconsolidação estimadas com a equação do intervalo de confiança a $95 \%$, considerada como sendo a região onde ocorre a compactação; (b) uma região onde as pressões de preconsolidação são menores do que as pressões de preconsolidação estimadas com a equação do intervalo de confiança a $95 \%$ e maiores do que as pressões estimadas com a equação $\sigma_{\mathrm{p}}=10^{(2,71-1,26 \mathrm{U})}$, sendo considerada a região onde há tendência em ocorrer a compactação do solo, e (c) uma região onde as pressões de preconsol idação são menores do que a pressão de preconsolidação estimada com a equação $\sigma_{\mathrm{p}}=10^{(2,71-1,26 \mathrm{U})}$, sendo considerada como a região onde não ocorre a compactação do solo.

Os valores das pressões de preconsolidação estimadas por meio da equação $\sigma_{\mathrm{p}}=99,39+0,39 \mathrm{RP}$ foram plotados na figura 6, obtendo-se a figura 7. Nesta figura, observa-se que as pressões de preconsolidação determinadas na linha de tráfego ocorreram na região de compactação do solo,

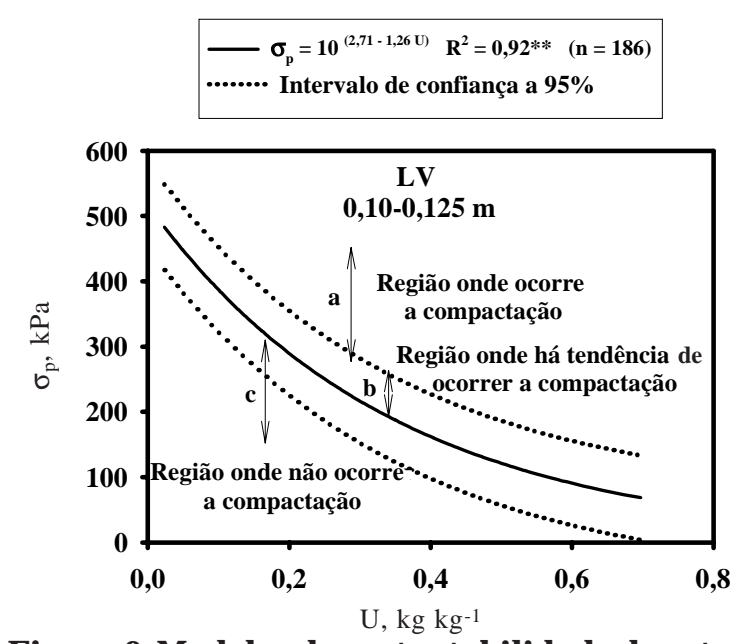

Figura 6. Model os de sustentabi lidade da estrutura para o LV, na profundidade de 0,10-0,125 m, com os critérios utilizados na identificação da compactação do solo.

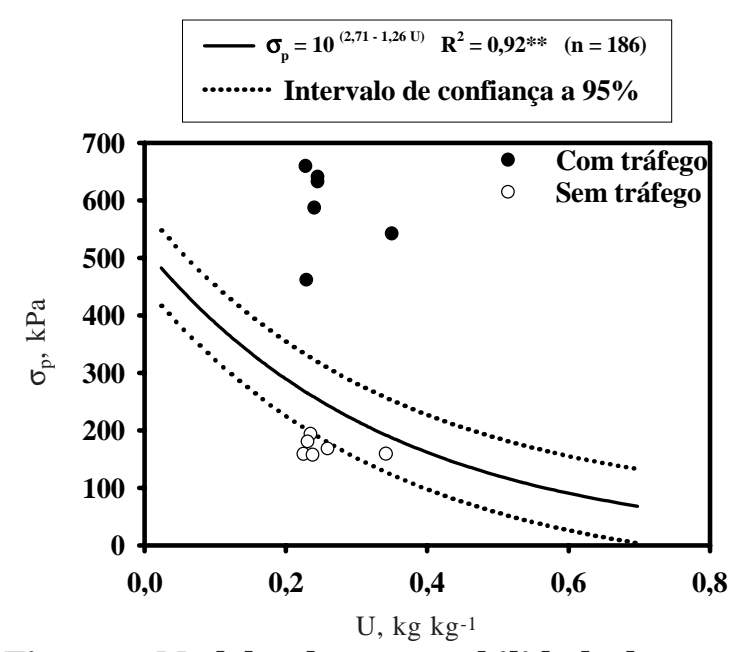

Figura 7. Model os de sustentabilidade da estrutura para o LV, na profundidade de 0,10-0,125 m. 
enquanto as pressões de preconsolidação determinadas em locais onde não houve tráfego situaram-se na região em que não ocorre compactação do solo, demonstrando que as pressões depreconsol idação estimadas por mei o da resistência à penetração podem ser usadas como um critério auxiliar na identificação da compactação do solo.

\section{CONCLUSÃO}

As pressões de preconsolidação podem ser determinadas em função da resistência à penetração do solo.

\section{LITE RATURA CITADA}

ANDERSON, T.C. \& LUKAS, R.G. Preconsolidation pressure predicted using Su/p'ratio. In: YONG, R.N. \& TOWNSEND, F.C., eds. Laboratory shear strength of soil. SYMPOSIUM OF THE ASTM. 1981. p.502-515. (Spec. Tech. Pub., 740)

BOWLES, J.E. Engineering properties of soils and their measurements. 3.ed. Auckland: McGraw-Hill, 1986. 218p.

BURMISTER, D. The application of controlled test methods in consolidation testing. In: Fifty-four annual meeting of the ASTM. SYMPOSIUM ON CONSOLIDATION TESTING OF SOILS. 1951. p.83-98. (Spec. Tech. Pub., 126)

CANILLAS, E.C. \& SALOKHE, V.M. A decision support system for compaction assessment in agricultural soils. Soil Till. Res., 65:221-230, 2002.

CASAGRANDE, A. The determination of the pre-consolidation load and its practical significance. In: CONFERENCE ON SOIL MECH. AND FOUND. ENG., PROC. of theICSMFE. Cambridge, 1936. Cambridge, 1936, v.3. p.60-64.

CULLEY, J .L.B. \& LARSON, W.E. Susceptibility to compression of a clay loam Haplaquoll. Soil Sci. Soc. Am. J., 51:562567, 1987.

DAY, P.R. Particle fractionation and particle size analysis. In: KLUTE, C.A., ed. Methods of soil analysis. Part 1, physical and mineralogical methods. 2.ed. Madison, American Society Agronomy, 1986. p.545-567. (Agron. Monogr., 9)

DEFOSSEZ, P. \& RICHARD, G. Models of soil compaction due totraffic and their evaluation. Soil Till. Res., 67:41-64, 2002.

DIAS J UNIOR, M.S. Compression of three soils under longterm tillage and wheel traffic. East Lansing, Michigan State University, 1994. 114p. (Tese de Doutorado)

DIAS J UNIOR, M.S. Avaliação de impactos de sistemas de colheita nas características físicas dos solos. Relatório Técnico do Projeto de Pesquisa das Regiões de Atuação da Cenibra. Período 1999 a 2002. Lavras, 2002. p.113.
DIAS J UNIOR, M.S.; FERREIRA, M.M.; FONSECA, S.; SILVA, A.R. \& FERREIRA, D.F. Avaliação da sustentabilidade estrutural dos solos em sistemas florestais na região de Aracruz - ES. R. Árvore, 23:371-380, 1999.

DIAS J UNIOR, M.S. \& PIERCE, F.J . A simple procedure for estimating preconsolidation pressure from soil compression curves. Soil Techn., 8:139-151, 1995.

DIAS J UNIOR, M.S. \& PIERCE, F.J . Revisão de literatura: O processo de compactação do sol o e sua modelagem. R. Bras. Ci. Solo, 20:175-182, 1996.

GUPTA, S.C.; HADAS, A. \& SCHAFER, R.L. Modeling soil mechanical behavior during compaction. In: LARSON, W.E.; BLAKE, G.R.; ALLMARAS, R.R; VOORHEES, W.B. \& GUPTA, S.C., eds. Mechanics and related process in structured agricultural soils. The Netherlands, Kluwer Academic Publishers, 1989. p.137-152. (NATO Applied Sciences 172)

GYSI, M. Compaction of an Eutric Cambisol under heavy wheel traffic in Switzerland: Field data and a critical state soil mechanics model approach. Soil Till. Res., 61:133-142, 2001.

HOLTZ, R.D. \& KOVACS, W.D. An introduction to geotechnical engineering. New J ersey, Prentice-Hall, 1981. 733p.

IMHOFF, S.; SILVA, A.P.; DIAS J UNIOR M.S. \& TORMENA, C.A. Quantificação de pressões para o crescimento das plantas. R. Bras. Ci. Solo, 25:11-18, 2001.

J OSE, B.T.; SRIDHARAN, A. \& ABRAHAM, B.M. Log-log method for determination of preconsolidation pressure. Geot. Tes. J ., 12:230-237, 1989.

KONDO, M.K. \& DIAS J UNIOR, M.S. Compressibilidade de três Latossolos em função da umidade e uso. R. Bras. Ci. Solo, 23:211-218, 1999.

LEBERT, M. \& HORN, R. A method to predict the mechanical strength of agricultural soils. Soil \& Till. Res., 19:274-286, 1991.

RAIJ , B. van. \& GUAGGIO, J.A. Métodos de análise de solo para fins de fertilidade. Campinas, Instituto Agronômico, 1983. 16 p. (Circular, 63)

SÄLLFORS, G. Preconsolidation pressure of soft high plastic clays. Gothenburg, Department of Geotechnical Engineering, 1975. (Tese de Doutorado)

SCHMERTMANN, J.H. The undisturbed consolidation behavior of clay. Trans. Soc. Civ. Eng., 120:1201-1233, 1955.

SNEDECOR, G.W. \& COCHARAN, W.G. Statistical methods. 8.ed. Ames, I owa State University Press, 1989. 503p.

TAYLOR, D.W. Fundamentals of soil mechanics. New York, J ohn Wiley \& Sons, 1948. 700p.

VARGAS, M. Introdução à mecânica dos solos. São Paulo, McGraw-Hill do Brasil, 1977. 509p. 